\title{
Smad4 controls bone homeostasis through regulation of osteoblast/osteocyte viability
}

\author{
Young Jae Moon ${ }^{1,2,5}$, Chi-Young Yun ${ }^{2,5}$, Hwajung Choi ${ }^{2}$, Sun-O Ka ${ }^{1}$, Jung Ryul Kim ${ }^{3,4}$, Byung-Hyun Park ${ }^{1}$ \\ and Eui-Sic Cho ${ }^{2}$
}

Regulation of osteoblast and osteocyte viability is essential for bone homeostasis. Smad4, a major transducer of bone morphogenetic protein and transforming growth factor- $\beta$ signaling pathways, regulates apoptosis in various cell types through a mitochondrial pathway. However, it remains poorly understood whether Smad4 is necessary for the regulation of osteoblast and osteocyte viability. In this study, we analyzed Smad44 ${ }^{O s}$ mice, in which Smad4 was subjected to tissue-specific disruption under the control of the 2.3-kb Col1a1 promoter, to understand the functional significance of Smad4 in regulating osteoblast/osteocyte viability during bone formation and remodeling. Smad44 ${ }^{0 s}$ mice showed a significant increase in osteoblast number and osteocyte density in the trabecular and cortical regions of the femur, whereas osteoclast activity was significantly decreased. The proliferation of osteoblasts/osteocytes did not alter, as shown by measuring 5 '-bromo-2'deoxyuridine incorporation. By contrast, the percentage of TUNEL-positive cells decreased, together with a decrease in the $\mathrm{Bax} / \mathrm{Bcl}-2$ ratio and in the proteolytic cleavage of caspase 3, in Smad44 ${ }^{O s}$ mice. Apoptosis in isolated calvaria cells from Smad44 ${ }^{0 s}$ mice decreased after differentiation, which was consistent with the results of the TUNEL assay and western blotting in Smad44 ${ }^{\text {os }}$ mice. Conversely, osteoblast cells overexpressing Smad4 showed increased apoptosis. In an apoptosis induction model of Smad44 ${ }^{\text {ss }}$ mice, osteoblasts/osteocytes were more resistant to apoptosis than were control cells, and, consequently, bone remodeling was attenuated. These findings indicate that Smad4 has a significant role in regulating osteoblast/osteocyte viability and therefore controls bone homeostasis. Experimental \& Molecular Medicine (2016) 48, e256; doi:10.1038/emm.2016.75; published online 2 September 2016

\section{INTRODUCTION}

Osteoblast and osteocyte viability have an important role in bone homeostasis. Many studies have found that signaling pathways involve crosstalk between osteoblasts and osteoclasts maintain the bone matrix depending on various physiologic and pathologic conditions. ${ }^{1-6}$ Osteoblast apoptosis, such as steroid-induced apoptosis and microgravity-induced apoptosis, stimulates osteoclastogenesis and bone resorption. ${ }^{7}$ Recent studies have demonstrated that receptor activator of nuclear factor- $\mathrm{KB}$ ligand (RANKL), which is released by apoptotic osteocytes, affects osteoclast activity and is essential for bone remodeling. ${ }^{8-12}$ Increasing osteoblast and osteocyte viability protects against osteoporosis induced by unloading, aging, sex steroid deficiency and excess glucocorticoids. ${ }^{10-12}$ Therefore, controlling osteoblast and osteocyte viability is essential for recovery from pathologic bone conditions.
Transforming growth factor- $\beta$ and bone morphogenetic protein (TGF- $\beta /$ BMP) signaling have critical roles in bone homeostasis. ${ }^{13-17}$ In the canonical pathway, each ligand transduces its signal by binding to a receptor, which forms a heterotetrameric complex. This complex phosphorylates intracellular, receptor-regulated Smads (R-Smads: Smad1, 2, 3, 5 and 8). Phosphorylated R-Smads combine with a common-mediator Smad, Smad4 and translocate to the nucleus, where they regulate target gene expression. Smad4 is a common mediator of TGF- $\beta /$ BMP signaling in bone homeostasis, unlike R-Smads. Smad1, 5 and 8 mediate BMP signaling, and Smad2 and 3 mediate TGF- $\beta$ signaling. In addition, Smad4 regulates apoptosis in a variety of cells. ${ }^{18-20}$ TGF- $\beta$ signaling triggers apoptosis in mouse mammary epithelial cells through a mitochondrial pathway. ${ }^{18}$ TGF- $\beta$ is also involved

\footnotetext{
${ }^{1}$ Department of Biochemistry, Chonbuk National University Medical School, Jeonju, Jeonbuk, Republic of Korea; ${ }^{2}$ Cluster for Craniofacial Development and Regeneration Research, Institute of Oral Biosciences, Chonbuk National University School of Dentistry, Jeonju, Jeonbuk, Republic of Korea; ${ }^{3}$ Department of Orthopaedic Surgery, Chonbuk National University Medical School, Jeonju, Jeonbuk, Republic of Korea and ${ }^{4}$ Research Institute of Clinical Medicine, Chonbuk National University Hospital, Jeonju, Jeonbuk, Republic of Korea

${ }^{5}$ These authors contributed equally to this work.

Correspondence: Professor E-S Cho, Cluster for Craniofacial Development and Regeneration Research, Institute of Oral Biosciences, Chonbuk National University School of Dentistry, 567 Baekje-Daero, Jeonju, Jeonbuk 54896, Republic of Korea.

E-mail: oasis@jbnu.ac.kr

Received 17 November 2015; revised 25 March 2016; accepted 5 April 2016
} 
a
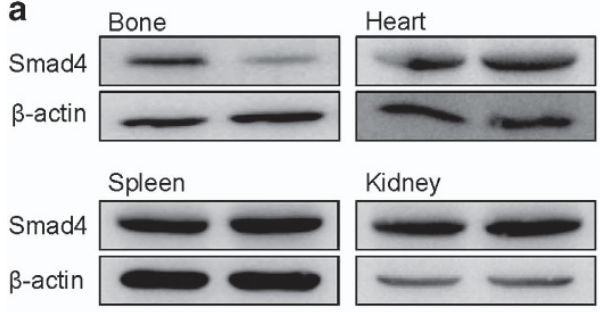

Control Smad4 4 Ss



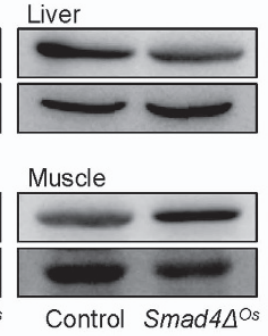

C
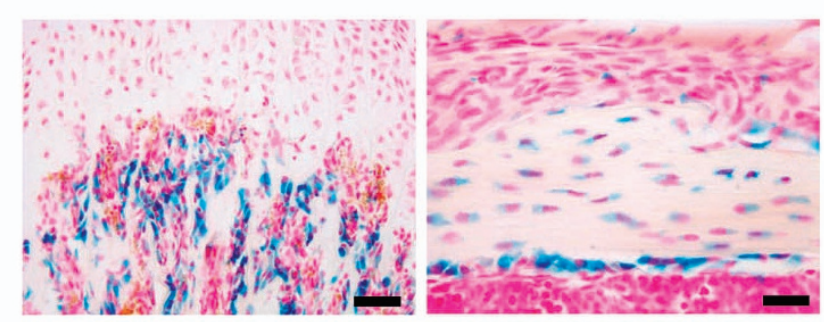

f
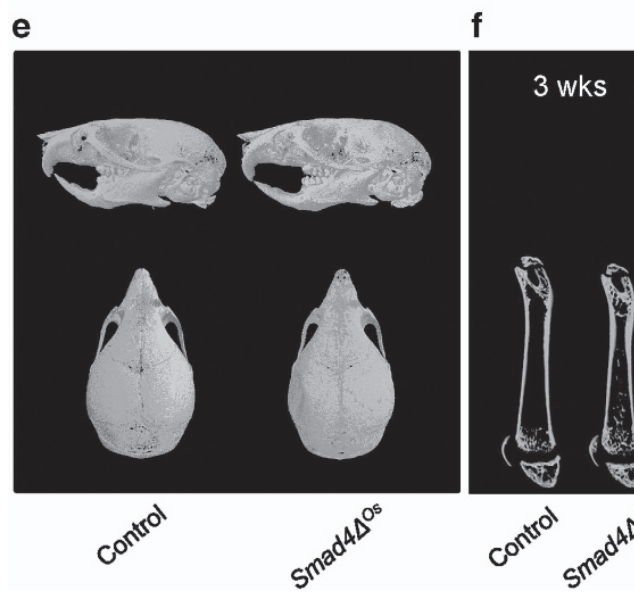

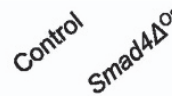

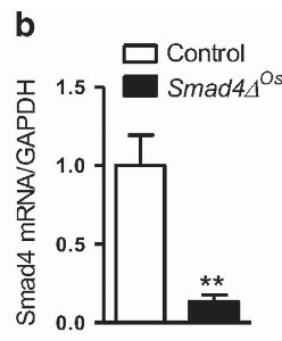

d

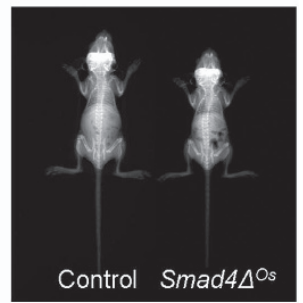

Control Smad $4 \triangle^{\text {Os }}$

Figure 1 Delayed growth in targeted Smad4-disrupted mice. (a) Disruption of Smad4 protein in Smad44 Os mice was verified by western blotting using total protein lysates from 3-week-old control and Smad44 ${ }^{O s}$ mouse femur, heart, liver, spleen, kidney and muscle. (b) Total RNA was isolated from the bones of each littermate mouse 3 weeks postnatally, and the mRNA level of Smad4 was determined using real-time RT-PCR analysis. (c) Characterization of Cre activity in 3-week-old Colla1-Cre:R26R double transgenic mice. The left panel indicates primary spongiosa in the distal femur. The right panel indicates cortical bone in the femur shaft. (d) The 3-week-old mice were imaged with simple radiography. (e) A 3D $\mu \mathrm{CT}$ reconstruction of the head in 3-week-old littermates. (f) $3 \mathrm{D} \mu \mathrm{CT}$ reconstruction of femora at 3 weeks, 6 weeks and 5 months of age. The reconstruction site was the center of the femur in the sagittal plane. Values are presented as the means \pm s.e.m. $(n=5) .{ }^{* *} P<0.01$ versus control. Scale bar (black), $50 \mu \mathrm{m}$ (c, left), $25 \mu \mathrm{m}$ (c, right). Scale bar (white), 2 mm.

in inducing apoptosis by affecting the Bax/Bcl-2 balance. ${ }^{21}$ Moreover, Smad-dependent BMP signaling induces osteoblast apoptosis via the mitochondrial pathway in mature osteoblast cells. ${ }^{22} \mathrm{We}$ focused on the role of Smad4 in apoptosis induction in bone because osteoblast apoptosis is known to be affected by both TGF- $\beta$ and BMP signaling.

However, Osx-Cre-mediated Smad4-disrupted mice show increased apoptosis of osteoblasts and osteocytes on the diaphysis. ${ }^{16}$ Therefore, it remains unclear whether Smad4 signaling is involved in osteoblast and osteocyte viability and apoptosis.

Here we generated tissue-specific Smad4-disrupted mice under the control of 2.3-kb Collal-Cre to investigate the roles of Smad4 signaling in the control of osteoblast and osteocyte viability.

\section{MATERIALS AND METHODS}

\section{Animals}

The Animal Welfare Committee of Chonbuk National University approved all animal procedures. Smad4-floxed allele (Smad4 $\left.4^{f / f}\right)$, Colla1-Cre and Rosa26 (R26R) reporter mice were described previously. ${ }^{23-25}$ To generate Colla1-Cre;Smad4 ${ }^{f l / f l}\left(S_{m a d} 4 \Delta^{\mathrm{Os}}\right.$ ) mice, Collal-Cre;Smad $4^{f / 1+}$ (control) mice were crossed with Smad $4^{f / f l}$ mice. Mouse genotypes were assessed by polymerase chain reaction analysis using previously described primers. ${ }^{23-25}$ To analyze Cre activity, Collal-Cre mice were crossed with $R 26 \mathrm{R}$ mice, and the femora of double-transgenic mice were processed for $X$-gal staining as described previously. ${ }^{26}$ 
Tissue preparation, immunohistochemistry, TRAP staining and TUNEL assay

Femora dissected from mice were fixed in $4 \%$ paraformaldehyde at $4{ }^{\circ} \mathrm{C}$ overnight. After rinsing with phosphate-buffered saline (PBS), the specimens were decalcified in 15\% EDTA/PBS for 3-5 weeks, dehydrated, embedded in paraffin and sectioned at a thickness of $5 \mu \mathrm{m}$. To acquire samples at the same position for each femur, we sectioned the bone on the basis of two points. The proximal point was the piriformis insertion site, and the distal point was the anterior crucial ligament origin site. The samples and sections were acquired at nearly the same location in the centers of the femora (Figure 1f). Slides of sections were stained with hematoxylin and eosin for histological analysis.

For immunostaining, sections were treated with 3\% hydrogen peroxide and incubated with rabbit polyclonal antibodies against caspase 3 (1:50; Abcam, Cambridge, MA, USA). A Histostain Plus rabbit primary (DAB) kit (Zymed Laboratories, San Francisco, CA, USA) was used according to the manufacturer's instructions. To detect osteoclasts, TRAP staining was performed with a TRAP staining kit (Sigma-Aldrich, St Louis, MO, USA). The number of osteoclasts in trabecular bone was counted by using microscopy. To detect apoptosis, a TACS 2 TdT-DAB In Situ Apoptosis Detection Kit (TREVIGEN, Gaithersburg, MD, USA) was used according to the manufacturer's instructions. The number of apoptotic osteocytes and total osteocytes in cortical bone were counted, and the percentage of apoptotic cells was calculated.

\section{Incorporation of 5'-bromo-2'deoxyuridine and cell proliferation assays}

To detect the extent of cell proliferation in the osteoblast and osteocyte populations, 5'-bromo-2'deoxyuridine (BrdU) labeling reagent (45 $\mathrm{\mu g} \mathrm{g}^{-1}$ body weight; Roche, Indianapolis, IN, USA) was injected intraperitoneally into mice at P10. Two hours after injection, their femora were dissected, embedded and sectioned in the mid-sagittal plane for immunodetection with a BrdU labeling and detection kit (Roche). For statistical analyses, three independent littermates were used in each of the studies.

\section{$\mathrm{X}$-ray and micro-computed tomography}

Mice were imaged with a high-resolution digital mammographic imager with a direct detection flat-panel array design (Mammomat Novation, Siemens AG Medical Solutions, Erlangen, Germany) and a flat-panel digital detector $(24 \mathrm{~cm}$; maximum matrix size $3328 \times 4096$; pixel size, $70 \mu \mathrm{m}$ ). All images were obtained with exposure settings of $34 \mathrm{kVp}$ and $110 \mathrm{~mA}$ at $\times 1.5$ magnification. For micro-computed tomography $(\mu \mathrm{CT})$, femora dissected from mice were scanned using a desktop scanner (1076 Skyscan Micro-CT, Skyscan, Kontich, Belgium) at $8-\mu \mathrm{m}$ resolution and analyzed with CTAn software (Skyscan). A global thresholding algorithm was applied at a constant threshold for all specimens. The threshold was the intensity (gray value) that corresponded to $\sim 50 \%$ of the average intensity of intact cortical bone. Trabecular lesions were analyzed in the distal metaphysis extending proximally $1.5 \mathrm{~mm}$ from the end of the growth plate. Cortical lesions were analyzed in the diaphyseal segment extending $1.5 \mathrm{~mm}$ proximally and distally from the midpoint between the femoral ends.

\section{Tail suspension}

Mechanical unloading was performed by tail suspension for 2 weeks (in 2-5-month-old female mice, $n=4$ per group). For tail suspension, a metal clip was attached to the tail with tape. The end of the clip was fixed to an overhead bar and the suspension was adjusted to maintain the mice at $\sim 30^{\circ}$ head down tilt. Grounded control littermates mice were caged under the same conditions without tail suspension. All mice were caged individually.

\section{Osteoblast isolation and mineralization}

We isolated osteoblasts from calvaria of control mice and $\operatorname{Smad} 4 \Delta^{\mathrm{Os}}$ mice at postnatal day 3. The periosteum and endosteum of individual calvaria were carefully removed, cleaned and thoroughly diced into small pieces, pooled for each pup, and digested in $1 \times$ PBS containing $4 \mathrm{mg} \mathrm{ml}^{-1}$ of collagenase Type II (Worthington, Lakewood, NJ, USA) at $37^{\circ} \mathrm{C}$ for $10 \mathrm{~min}$. The initial digestion was discarded, and the supernatants from the second and third sequential digestions at $37^{\circ} \mathrm{C}$ were collected. Cells were collected by centrifugation and resuspended in $\alpha$ MEM supplemented with $100 \mathrm{U} \mathrm{ml}^{-1}$ penicillin, $100 \mu \mathrm{g} \mathrm{ml}^{-1}$ streptomycin and $10 \%$ fetal bovine serum. Primary osteoblasts were seeded on 100-mm culture dishes at an initial density of $1 \times 10^{5}$ cells. For mineralization, cells were incubated for 21 days with $10 \mathrm{~mm}$ $\beta$-glycerophosphate and $50 \mu \mathrm{g} \mathrm{ml}^{-1}$ ascorbic acid. The culture medium was replaced every 2-3 days. The mineralization matrix was stained with $40 \mathrm{~mm}$ alizarin red solution at day 14 after the initial mineralization.

\section{Cell culture and transient transfection of plasmid}

7F2 cells (ATCC, Manassas, VA, USA) were cultured in DMEM supplemented with $100 \mathrm{U} \mathrm{ml}^{-1}$ penicillin, $100 \mu \mathrm{g} \mathrm{ml}^{-1}$ streptomycin and $10 \%$ fetal bovine serum. Transient transfection was performed using Lipofectamine 2000 (Invitrogen, Carlsbad, CA, USA) according to the manufacturer's instructions. Briefly, $\sim 80 \%$ confluent $7 F 2$ cells were transfected with $5 \mu \mathrm{g}$ of pRK-DPC4-Flag (Smad4) and the empty vector (Empty). After $24 \mathrm{~h}$, the cells were harvested in a protein extraction solution (Pro-Prep; Intron Biotechnology, Seoul, Korea) with protease and phosphatase inhibitors.

\section{Mineralization and detection of apoptosis}

$7 \mathrm{~F} 2$ cells were seeded on $60-\mathrm{mm}$ culture dishes at an initial density of $2 \times 10^{5}$ cells. After transfection of Smad4 and empty vector, the cells were incubated for 5-7 days with $10 \mathrm{~mm} \beta$-glycerophosphate and $50 \mu \mathrm{g} \mathrm{ml}^{-1}$ ascorbic acid. The culture medium was replaced every 2-3 days. Apoptosis was determined using an APOPercentage apoptosis assay kit (Biocolor Ltd, Belfast, Northern Ireland) on day 6 after initial mineralization. Digital images of APOPercentage dye-labeled cells, appearing bright pink against a white background under a light microscope, were used to detect the apoptotic cells. To quantify apoptosis, cells were incubated with a 1:100 dilution of the dye for 5 min. After staining, cells were washed with PBS, and the dye within the labeled cells was subsequently released into the supplied dye release reagent. The contents of each dish were transferred to a 96-well flat-bottomed plate and the absorbance was read at $550 \mathrm{~nm}$ using a microplate reader.

\section{Quantitative real-time PCR with reverse transcription analysis}

Femora of control and mutant mice were dissected free of soft tissue, and the bone marrow was flushed away. Femora frozen in liquid nitrogen were crushed with a mortar and pestle. Total RNA was extracted with TRIzol reagent (Invitrogen) according to the manufacturer's instructions. RNA from extracted tissues was precipitated with isopropanol and dissolved in DEPC-treated distilled water. cDNA was synthesized from random hexamer primers from 
a
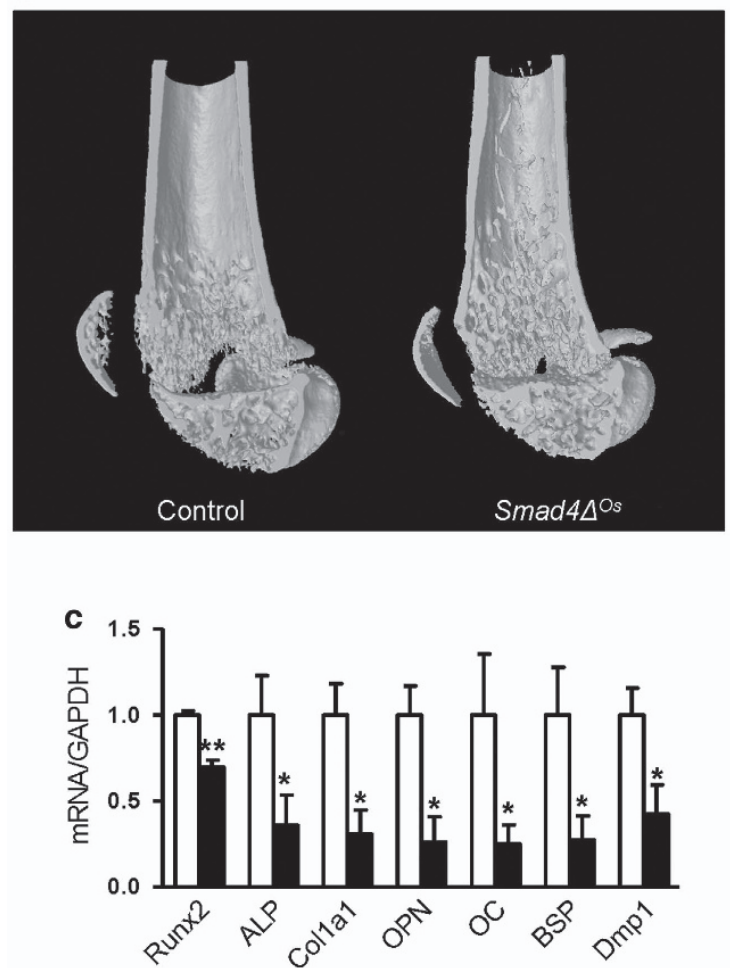

b


e

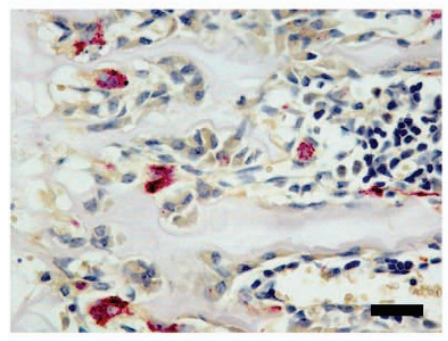

Control

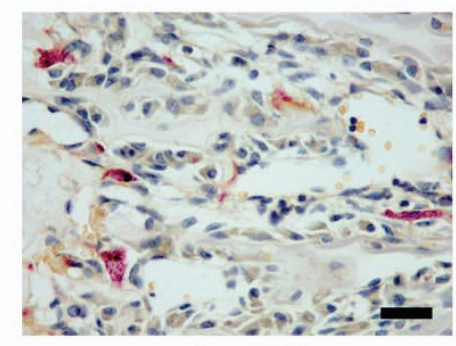

Smad $4 \triangle \mathrm{Os}$ f

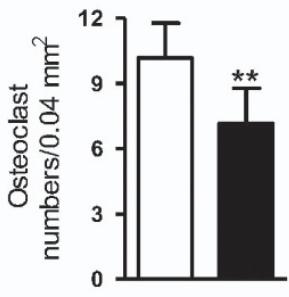

Figure 2 Increased bone mass in Smad4 $\Delta^{O S}$ mice. (a, b) 3D $\mu \mathrm{CT}$ reconstructions of distal femora of 3-week-old littermates and hematoxylin and eosin stain. (c) Total RNA was isolated from the bones of each littermate mouse at 3 weeks postnatal, and the mRNA levels of markers were determined by real-time RT-PCR analysis. Changes in the bone formation markers Runx2, Alp, Col1a1, OPN, OC, $B S P$ and Dmp1. (d) Changes in the bone resorption markers Rankl, Opg, Trap and Ctsk. (e, f) TRAP staining in primary spongiosa of distal femora of 3-week-old mice, and counted osteoclasts per unit area. Values are presented as the means \pm s.e.m. $(n=5)$. ${ }^{*} P<0.05$ and ${ }^{* *} P<0.01$ versus control. Scale bars, $400 \mu \mathrm{m}(\mathbf{b}), 25 \mu \mathrm{m}(\mathbf{e})$.

first-strand cDNA synthesis kits according the manufacturer's instructions (Applied Biosystems, Foster City, CA, USA). Specific primers were designed (Supplementary Table S1) using Primer Express software (Applied Biosystems). GAPDH was used as an invariant control. PCR reactions, data quantification and analysis were performed. Samples from five mice from each group were analyzed, and RNA from individual mice was analyzed separately.

\section{Western blotting}

Equal amounts of protein extracts were separated on $10 \%$ SDS-polyacrylamide gel electrophoresis and blotted onto polyvinyl difluoride membrane. The blots were probed overnight with primary antibodies as follows: mouse antibodies against Bax (Enzo Life Sciences, Farmingdale, NY, USA) and $\beta$-actin (Santa Cruz Biotechnology, Inc., Santa Cruz, CA, USA) and rabbit antibodies against Smad4 (Abcam), Bcl-2 (Bioworld Technology, Inc., St Louis Park, MN, USA), cleaved caspase 3 (Cell Signaling Technology, Danvers, MA, USA) and collagen I (Abcam).

\section{Statistical analysis}

One-way analysis of variance was used for multiple comparisons, and Student's $t$-test was used to determine significant differences between two sets of data. Differences were considered significant at $P<0.05$. All data were analyzed using SPSS statistical software (version 16.0; SPSS, Chicago, IL, USA).

\section{RESULTS}

Osteoblast/osteocyte-specific disruption of Smad4 leads to delayed growth in modeling state

We measured Smad4 protein expression in the major organs of Smad4 $4{ }^{O s}$ mice (Figure 1a). Smad4 expression was decreased 
in bone but was not altered in other major organs. In addition, the Smad4 mRNA level was significantly reduced in the bone of $S m a d 4 \Delta^{O s}$ mice (Figure 1b). We analyzed Cre activity using $X$-gal staining in Col1a1-Cre;R26R mice (Figure 1c). $\beta$-galactosidase activity was observed in most osteoblasts in the trabecular region and in all endosteum-lining osteoblasts and osteocytes in cortical bone. Smad $4 \Delta^{\mathrm{Os}}$ mice were appeared normal at birth and had body sizes and weights similar to those of littermate controls (data not shown). Smad $4 \Delta^{O s}$ mice exhibited slightly smaller bodies and decreased femur length at 3 weeks (Figure 1d and f), but spontaneous fracture, severe bone abnormalities and craniofacial bone defects were not observed (Figure 1d and e). Although Smad $4 \Delta^{O s}$ mice had slightly smaller bodies than controls at 3 weeks, these differences decreased gradually with age (Supplementary Figure S1). Furthermore, trabecular bone mass of Smad $4 \Delta^{\mathrm{Os}}$ mice increased slightly at 3 weeks, and these changes were greater at 6 weeks and 5 months (Figure 1f).

Smad4 regulates bone formation and affects bone resorption In the $\mu \mathrm{CT}$ analysis of trabecular bone in the distal femur, the ratio of bone volume to total tissue volume (BV/TV) was $\sim 2$-fold higher in Smad4d ${ }^{\mathrm{Os}}$ mice than in control mice at 3 weeks. Trabecular separation (Tb.Sp) was significantly lower in Smad4 $4{ }^{O s}$ mice than in control mice (Figure 2a and Table 1). Hematoxylin and eosin staining showed that the trabecular bone of the epiphysis and metaphysis in Smad4 $4 \Delta^{O s}$ mice was thicker than that in control mice at 3 weeks (Figure $2 \mathrm{~b}$ ). Because changes in bone volume are associated with bone homeostasis, we evaluated mRNA levels of genes that are markers of bone formation and resorption. The mRNA expression levels of pre-osteoblast marker genes, Runx2, alkaline phosphatase $(A L P)$, collagen (Colla1) and osteopontin $(O P N)$, were significantly decreased in Smad44 ${ }^{\mathrm{Os}}$ mice. In addition, the expression levels of mature osteoblast markers, namely, osteocalcin $(O C)$ and bone sialoprotein $(B S P)$, were reduced in Smad $4 \Delta^{O s}$ mice. An early osteocyte marker gene, dentin matrix protein 1 (Dmp1), was also decreased in Smad $4 \Delta^{\mathrm{Os}}$ mice (Figure 2c). These results suggest that bone mineralization was reduced at all osteoblast/osteocyte stages in $\operatorname{Smad} 4 \Delta^{O s}$ mice.

Table 1 Morphometric indices in trabecular bone of the distal femur of control and Smad44 ${ }^{\text {Os }}$ at 3-week old

\begin{tabular}{lcc}
\hline & Control & Smad44 ${ }^{\text {Os }}$ \\
\hline BV $\left(\mathrm{mm}^{3}\right)$ & $0.186 \pm 0.071$ & $0.367 \pm 0.175$ \\
BV/TV $(\%)$ & $7.15 \pm 3.77$ & $14.66 \pm 3.85^{\mathrm{a}}$ \\
Tb.Th $(\mathrm{mm})$ & $0.049 \pm 0.005$ & $0.054 \pm 0.006$ \\
Tb.N $\left(\mathrm{mm}^{-1}\right)$ & $1.42 \pm 0.64$ & $3.33 \pm 0.14$ \\
Tb.Sp $(\mathrm{mm})$ & $0.337 \pm 0.037$ & $0.184 \pm 0.054^{\mathrm{a}}$
\end{tabular}

Abbreviations: BV, bone volume; Tb.N, trabecular number; Tb.Sp, trabecular separation; Tb.Th, trabecular thickness; TV, tissue volume.

Values represent mean \pm s.d. for four animals in each group.

a $P<0.05$ versus control.
Expression of the osteoclast bone resorption marker, tartrate-resistant acid phosphatase (Trap), decreased significantly in $S m a d 4 \Delta^{O s}$ mice compared with control mice. In addition, the expression of cathepsin $\mathrm{K}$ (Ctsk), a lysosomal enzyme essential for osteoclastic bone resorption, was reduced, although not significantly (Figure 2d). We also measured the expression levels of receptor activator of NFkB ligand (Rankl) and osteoprotegerin $(\mathrm{Opg})$, which are secreted by osteoblasts/ osteocytes to regulate osteoclastogenesis. ${ }^{1,2}$ RANKL expression decreased significantly by $\sim 30 \%$, but Opg expression did not change (Figure 2d). Consistent with these data, TRAP-positive multinucleated osteoclasts were reduced on the trabecular bone surface of the distal femur in Smad4 $4 \Delta^{\mathrm{Os}}$ mice (Figure 2e and f). These results indicate that deleting Smad4 in osteoblasts/ osteocytes decreased osteoclastogenesis. Although the expression of bone mineralization markers was reduced in Smad $4 \Delta^{O s}$ mice, trabecular bone mass increased due to decreased osteoclastogenesis.

\section{Smad4 is essential for osteoblast/osteocyte viability}

The density of osteoblasts in the primary spongiosa, endosteum-lining osteoblasts and osteocytes in cortical bone increased significantly at 3 weeks postnatal in $\mathrm{Smad} 4 \Delta^{\mathrm{Os}}$ mice (Figures $3 \mathrm{a}$ and $\mathrm{b}$ ). These changes matched Colla1-Cre activity (Figure 1c). Although these differences in cell density were not observed at 3 days postnatal, the density of osteocytes continued to increase in adult mice, and the density of osteoblasts in the primary spongiosa increased until growth stopped (Supplementary Figures S2a and S2b). A change in proliferation and apoptosis could alter cell density and cell viability. To examine differences in proliferation between the control and Smad44 ${ }^{\mathrm{Os}}$ mice, BrdU labeling was performed on postnatal day 10 mice. BrdU-labeled cells were not different between control and Smad $4 \Delta^{O s}$ mice on trabecular osteoblasts and cortical osteocytes (Figure $3 \mathrm{c}$ and $\mathrm{d}$ ). To investigate differences in apoptosis in Smad $4 \Delta^{O s}$ mice, TUNEL staining and western blotting was performed on bone tissue in 3 weeks postnatal mice. The percentage of TUNEL-positive cells decreased significantly in Smad44 ${ }^{\mathrm{Os}}$ mice (Figure 3e). To measure apoptosis levels in bone tissue, Bax (a pro-apoptotic factor), Bcl-2 (an anti-apoptotic factor) and cleaved caspase 3 (an executioner caspase), which are integral to the mitochondrial apoptosis pathway, were examined by western blotting. The $\mathrm{Bax} / \mathrm{Bcl}-2$ ratio and protein level of cleaved caspase 3 were decreased in Smad44 ${ }^{O s}$ mice (Figure $3 \mathrm{f}$ ). These data indicate that Smad4 regulates osteoblast/osteocyte apoptosis and viability through the mitochondrial apoptosis pathway.

\section{Smad4 regulates osteoblast apoptosis and mineralization in vitro}

To observe the effect of Smad4 on osteoblast apoptosis in vitro, we extracted osteoblasts from calvaria in control mice and Smad $4 \Delta^{O s}$ mice at postnatal day 3 and induced differentiation using ascorbic acid and $\beta$-glycerophosphate. Previous studies demonstrated that osteoblast differentiation induced osteoblast 
a

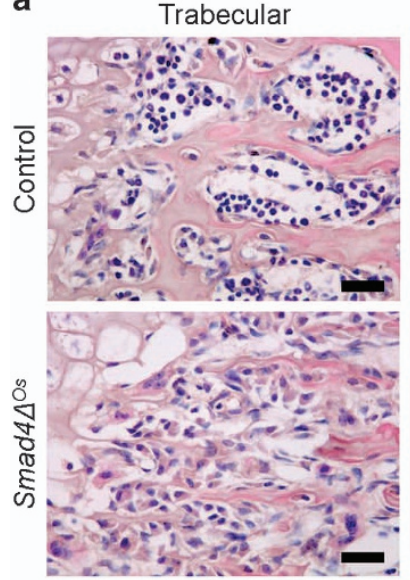

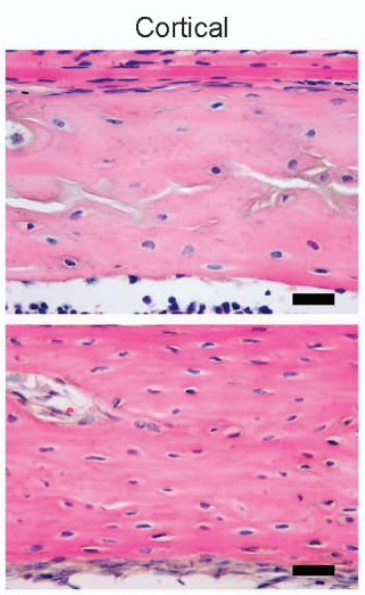

Cortical

c

Trabecular

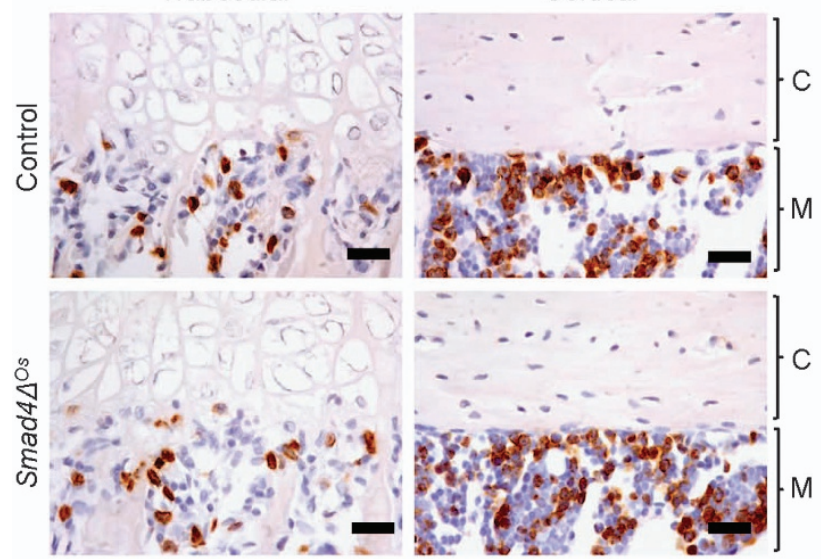

e

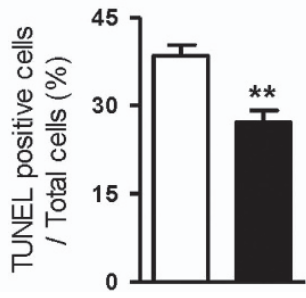

b

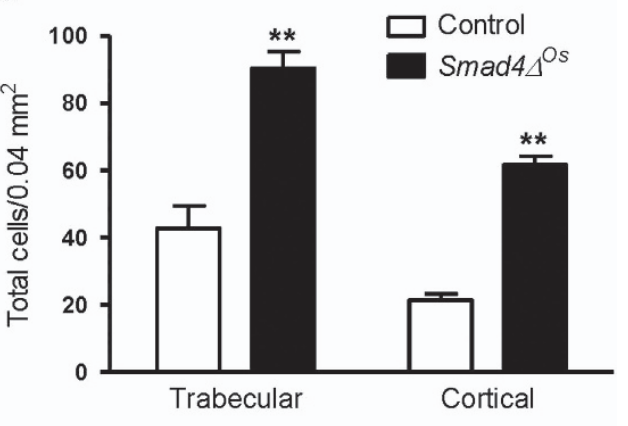

d



f

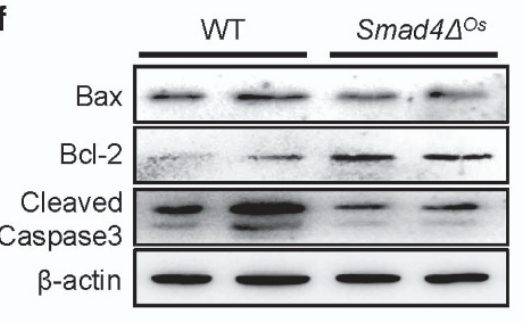

Figure 3 Regulation of osteoblast/osteocyte viability by Smad4. (a, b) Histological examination of primary spongiosa and cortical bone of distal femur stained with hematoxylin and eosin in control and Smad44 ${ }^{\text {Os }}$ mice at 3 weeks of age and counted osteoblasts and osteocytes per unit area. (c) Proliferation was evaluated using a BrdU labeling and detection kit at P10. (d) BrdU-labeled cells in trabecular osteoblasts and cortical osteocytes were counted per unit area. (e) Apoptotic osteocytes detected by TUNEL staining at 3 weeks of age. TUNEL-positive cells were counted and expressed as a percentage of all osteocytes per unit area. (f) Protein extracts of prepared bone and the levels of apoptosis-related proteins were examined by western blotting. $\mathrm{C}$, cortical bone, $\mathrm{M}$, bone marrow. Values are presented as the means \pm s.e.m. $(n=5) .{ }^{* *} P<0.01$ versus control. Scale bar, $25 \mu \mathrm{m}(\mathbf{a}, \mathbf{c})$.

apoptosis. ${ }^{27,28}$ After 3 weeks of mineralization, the protein level of Smad4 was decreased in primary osteoblasts from Smad4 4 Os mice (Figure 4b). In addition, mineralization of primary osteoblasts from Smad4 $\Delta^{\mathrm{Os}}$ mice decreased as determined by collagen I (Figure 4b) from western blotting and alizarin red staining (Figure 4a). Similar to the results of TUNEL staining and western blotting (Figures $3 \mathrm{e}$ and $\mathrm{f}$ ), the protein levels of proapoptotic caspase 3 and Bax decreased, whereas the expression of antiapoptotic Bcl-2 increased in primary osteoblasts from Smad4 $\Delta^{O s}$ mice (Figure $4 \mathrm{~b}$ ). These results showed that osteoblasts from Smad4 $\Delta^{O s}$ mice were functionally inadequate for mineralization and that apoptosis was suppressed. Consistent with previous reports ${ }^{27,28}$, these results suggest that immature mineralization affects apoptotic cell death in osteoblasts. To confirm the role of Smad4 on the apoptosis of osteoblasts, 7F2 cells, mature osteoblast cells derived from bone marrow, were transfected with empty vector or Smad4. Overexpressed Smad4 was demonstrated by western blotting (Figure 4c). The Bax/Bcl-2 ratio and protein level of cleaved caspase 3 were increased in 7F2 cells with 

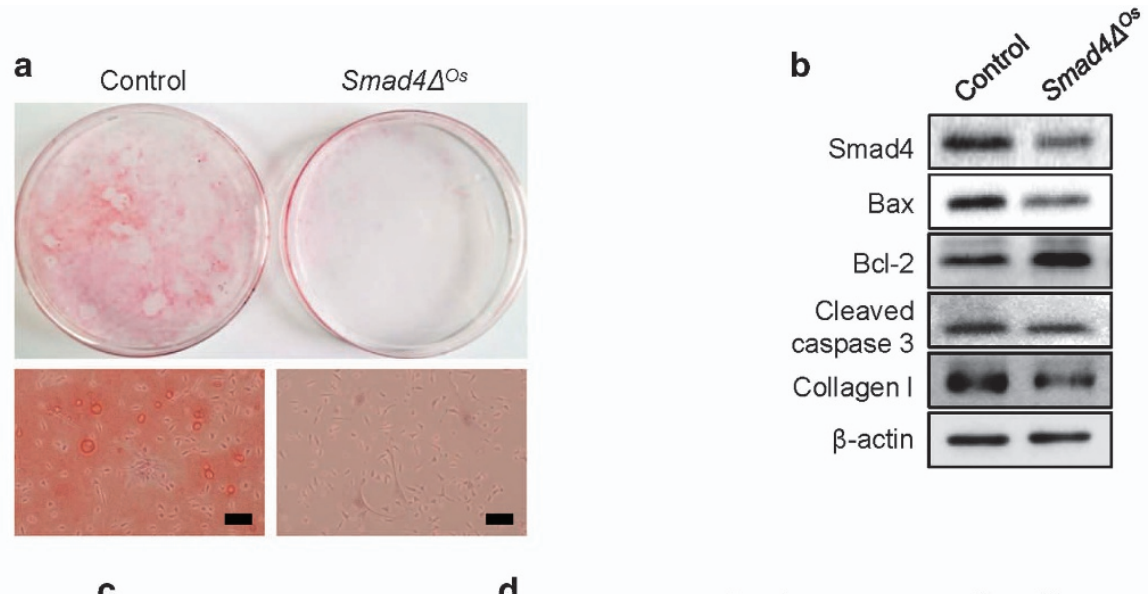

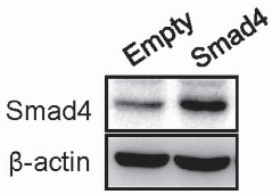

d

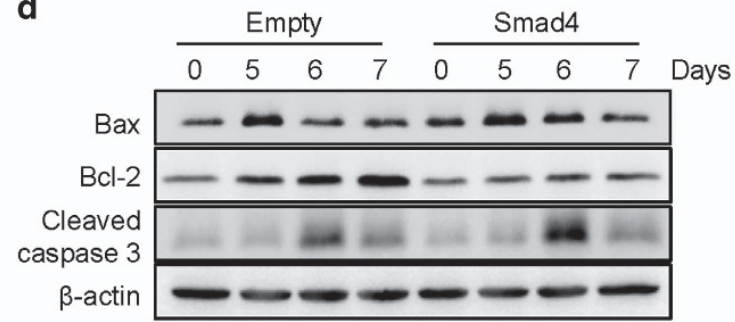

f

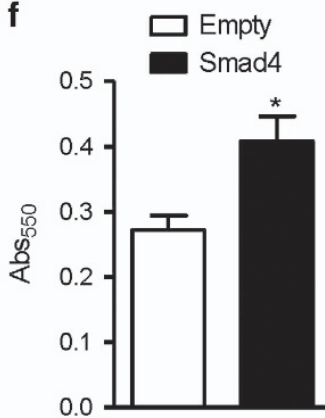

Figure 4 The effect of Smad4 on apoptosis of osteoblasts in vitro. (a, b) Primary osteoblasts were isolated from calvaria of control mice and $S m a d 4 \Delta^{O s}$ mice at postnatal day 3. (a) Isolated cells were stained with alizarin red solution on day 14 after initial mineralization. (b) The levels of apoptosis-related proteins were examined by western blotting on day 21 after initial mineralization. (c-f) 7F2 cells were transfected with either empty vector or Smad4 for $24 \mathrm{~h}$ and then treated with $10 \mathrm{~mm} \beta$-glycerophosphate and $50 \mu \mathrm{g} \mathrm{ml} \mathrm{I}^{-1}$ ascorbic acid. (c) Smad4 overexpression was analyzed by western blotting after transfection. (d) The levels of apoptosis-related proteins were examined by western blotting at the indicated time points after mineralization. (e) Apoptosis in 7F2 cells was assessed using an APOPercentage apoptosis assay kit. Apoptotic cells appear bright pink. (f) The absorbance of the accumulated APOPercentage dye was measured with a spectrophotometer at $550 \mathrm{~nm}$. Values are presented as the means \pm s.e.m. $(n=3)$. ${ }^{*} P<0.05$ versus empty vector. Scale bar, $100 \mu \mathrm{m}$.

overexpressed Smad4 after differentiation (Figure 4d). Furthermore, compared with the empty vector group, many Smad4-transfected cells underwent apoptotic changes, as determined using the APOPercentage apoptosis assay kit (Figures $4 \mathrm{e}$ and $\mathrm{f}$ ).

\section{Disruption of Smad4 resists bone loss caused by unloading induced apoptosis}

To determine whether Smad4 is important for controlling osteoblast/osteocyte viability in a pathologic state, we tailsuspended Smad $4 \Delta^{O s}$ and control littermates for 2 weeks to induce apoptosis. In control mice, the bone volume, BV/TV and cortical thickness decreased significantly due to unloadinginduced apoptosis. In addition, the total porosity significantly increased in the cortical bone of tail-suspended control mice (Figure 5a and Table 2). However, the bone volume, $\mathrm{BV} / \mathrm{TV}$ and cortical thickness were not significantly decreased and total porosity was not significantly induced in Smad4s ${ }^{O s}$ mice (Figure 5a and Table 2). Consistent with the protective effect on bone mass, the density of endosteum-lining osteoblasts/osteocytes in Smad4 $4{ }^{O s}$ mice was not changed after unloading (Figures 5b and c). By contrast, the density of endosteum-lining osteoblasts and osteocytes was significantly decreased in control mice after unloading (Figures $5 b$ and c). Furthermore, TUNEL-positive osteocytes showed a pattern similar to that of the cell density. TUNEL-positive osteocytes increased significantly in tail-suspended control mice but not in similarly treated Smad4 $\Delta^{O s}$ mice (Figures $5 \mathrm{~d}$ and e). 
a

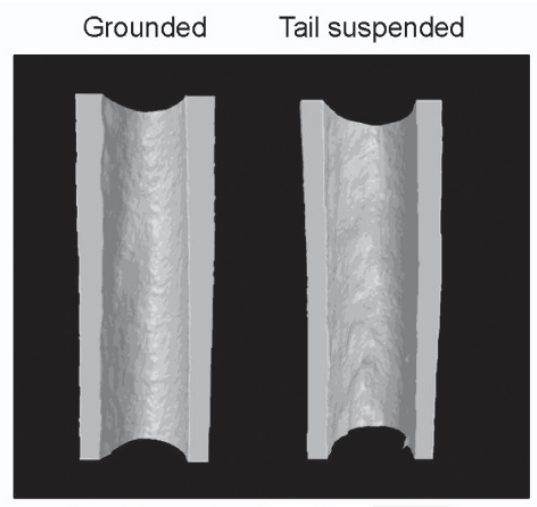

Control

b
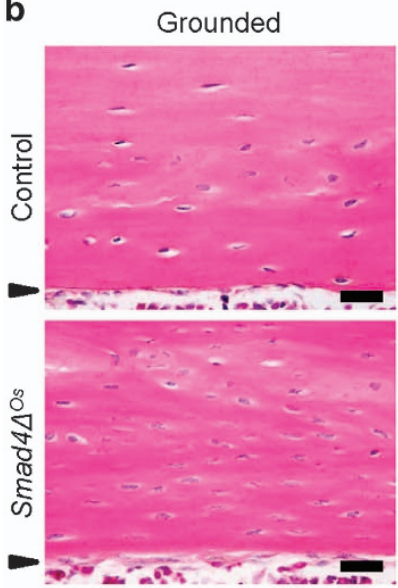

d
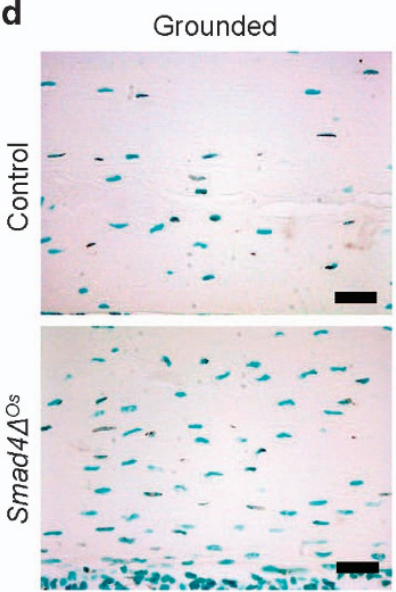

Tail suspended

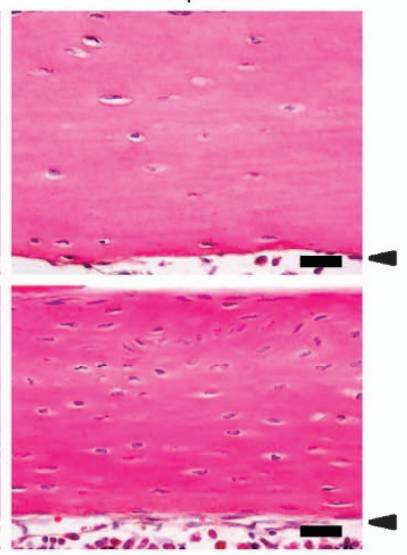

Tail suspended



$\mathrm{Smad} 4 \triangle^{\mathrm{OS}}$

C

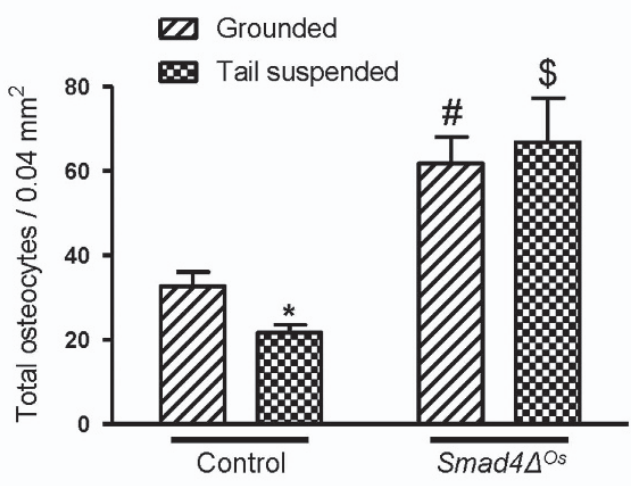

e
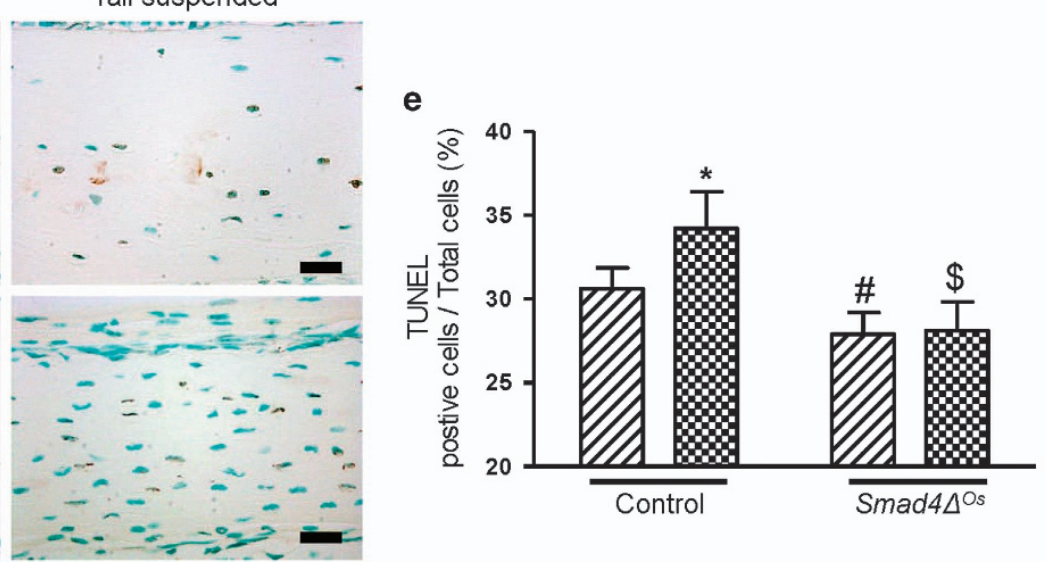

Figure 5 Protection against unloading-induced reduction of bone mass and apoptosis in Smad44 ${ }^{\text {Os }}$ mice. (a) Five-month-old control and $S$ mad4 $4 \Delta^{O s}$ mice were subjected to skeletal unloading by tail suspension or kept on the ground for 2 weeks. Three-dimensional $\mu C T$ reconstructions of diaphyseal femora. (b) Histological examination of cortical femora stained with hematoxylin and eosin in control and Smad4 $\Delta^{\text {Os }}$ mice at 2 months of age following 2 weeks of unloading by tail suspension or staying on the ground. Arrowheads indicate endosteum-lining osteoblasts. (c) Osteocytes were counted per unit area. Oblique line bars indicate grounded mice, and dotted bars indicate tail-suspended mice during 2 weeks of treatment. (d) Apoptotic osteocytes detected by TUNEL staining. (e) TUNEL-positive cells were counted and expressed as a percentage of all osteocytes per unit area. Hatched bars indicate grounded mice, and dotted bars indicate tail-suspended mice. Values are presented as the means \pm s.e.m. $(n=4)$. ${ }^{*} P<0.05$ versus control grounded; ${ }^{\#} P<0.01$ versus control grounded; $\$ P<0.01$ versus control tail-suspended. Scale bar, $25 \mu \mathrm{m}(\mathbf{b}, \mathbf{d})$. 
Table 2 Morphometric indices in cortical bone of the diaphyseal femur of control and Smad44 ${ }^{0 s}$ after 2 weeks of normally grounded or tail suspended at 2-5 months old

\begin{tabular}{|c|c|c|c|c|}
\hline \multirow[b]{2}{*}{ Bone structure parameter } & \multicolumn{2}{|c|}{ Control } & \multicolumn{2}{|c|}{ Smad44 Os } \\
\hline & Grounded & Tail suspended & Grounded & Tail suspended \\
\hline $\mathrm{BV}\left(\mathrm{mm}^{3}\right)$ & $0.506 \pm 0.074$ & $0.412 \pm 0.085$ & $0.532 \pm 0.017$ & $0.539 \pm 0.023$ \\
\hline BV/TV (\%) & $67.61 \pm 4.15$ & $63.37 \pm 2.80^{\mathrm{a}}$ & $72.15 \pm 3.53^{\mathrm{a}}$ & $71.24 \pm 5.73^{b}$ \\
\hline Co. thickness (mm) & $0.247 \pm 0.027$ & $0.205 \pm 0.039^{a}$ & $0.299 \pm 0.032^{\mathrm{a}}$ & $0.281 \pm 0.076^{b}$ \\
\hline Total porosity (\%) & $32.69 \pm 4.59$ & $37.21 \pm 2.49^{\mathrm{a}}$ & $27.84 \pm 3.53^{a}$ & $29.66 \pm 5.89^{b}$ \\
\hline
\end{tabular}

Abbreviations: BV, bone volume; Co, cortical; TV, tissue volume.

Values represent mean \pm s.d. for four animals in each group.

a $P<0.05$ versus control grounded.

${ }^{\mathrm{b}} \mathrm{P}<0.05$ versus control tail suspended.

Using immunohistochemical staining, caspase 3 was localized in endosteal osteoblasts and osteocytes of tail-suspended control mice. In addition, empty lacunae were present in the cortical bone of these mice. However, caspase 3 expression was absent in endosteal osteoblasts and osteocytes of tail-suspended Smad $4 \Delta^{\mathrm{Os}}$ mice (Supplementary Figure S3). These data indicate that disruption of Smad4 protects against bone loss and apoptosis resulting from unloading.

\section{DISCUSSION}

In this study, we investigated the functional significance of Smad4 in regulating osteoblast/osteocyte viability during bone formation and remodeling. Colla1-Cre-mediated disruption of Smad4 in osteoblasts/osteocytes reduced osteoblast/osteocyte apoptosis and decreased osteoclast activity. In addition, in a model of induced apoptosis, Smad44 $\Delta^{\mathrm{Os}}$ osteoblasts/osteocytes were more resistant to apoptosis than were controls. Consequently, bone remodeling was suppressed, and osteoclast activity was decreased.

It has previously been reported that Smad4 affects bone homeostasis. ${ }^{16}$ However, the bone phenotypes described in this previous study were somewhat different from our results. The authors showed that mice with Osx-Cre-mediated Smad4 disruption had severe growth retardation, hypomineralization and multiple rib fractures. By contrast, in our study, Smad4-disrupted mice exhibited delayed growth in the modeling state; however, they mostly recovered with age and did not exhibit craniofacial bone defects or rib fractures (Figures 1d and e). Furthermore, adult mutant mice demonstrated greater cortical thickness and less porosity than control mice (Table 2). These differences may be the result of several factors. First, the phenotypic differences may have been caused by Cre-activity. Colla1-Cre activity was observed in mature osteoblasts, located in the trabecular bone and endosteum, and osteocytes. Furthermore, Smad4 expression was not changed in other major organs, as evaluated by western blotting (Figures 1a and c). However, Osx-Cre activity was observed not only in osteoblasts and osteocytes but also in precursor osteoblasts, chondrocytes, stromal cells, adipocytes and perivascular cells in the bone marrow. Furthermore, Osx-Cre targeted tissues in addition to the skeleton, including gastric and intestinal epithelia. ${ }^{29}$ Second, osteoblast functions vary according to the state of cell maturation. In osteoprogenitor cells, treatment with BMP2 had only small effects on apoptotic signs. However, in mature osteoblast cells, BMP2 resulted in a robust increase in apoptosis through the Smad pathway. ${ }^{22}$ Therefore, Smad4 disruption in mature osteoblasts can increase resistance to apoptosis. Third, Osx-Cre mice have their own phenotypes. Several studies reported that Osx-Cre mice exhibit multiple bone defects and that these mice have delayed and reduced calvarial mineralization. Furthermore, fracture callus spots were frequently observed on multiple bone lesions. ${ }^{30-32}$

Although Smad4 is a common mediator of both TGF- $\beta$ and BMP signaling, the phenotypes of the Smad4t ${ }^{\mathrm{Os}}$ mice were more similar to those of mutants with disrupted BMP signaling. In Bmprla-disrupted mice, the numbers of osteoblasts and osteocytes increased with an increase in bone mass. ${ }^{15,33}$ In these mice, osteoclastogenesis, as well as the bone formation rate, were reduced, resulting in increased bone mass. By contrast, T TRRI conditional knockout mice exhibited not only increased bone formation but also increased osteoclastogenesis, similar to observations in active PTHIR mice. $^{34}$ In calvarial osteoblasts, treatment with TGF- $\beta$ decreased Runx2 expression and alkaline phosphatase activity. ${ }^{16,35}$ The authors suggested that TGF- $\beta$-activated Smad3 regulates osteoblast gene expression by repressing Runx2 transcription. ${ }^{35}$ However, treatment with BMP2 increased Runx2 expression and ALP activity in osteoblasts. ${ }^{16}$ These results indicate that TGF- $\beta$ and BMP signaling have different roles in Runx2 expression and ALP activity in osteoblasts. On the basis of our data, disruption of Smad4 in osteoblasts/osteocytes reduced the expression of Runx2 and ALP (Figure 2c). Therefore, the role of Smad4 in osteoblasts/osteocytes may be more affected by BMP signaling than TGF- $\beta$ signaling.

Although Smad4 is known to increase apoptosis in a variety of cells, it remains unclear whether Smad4 is involved in regulating the viability of mature osteoblasts and osteocytes in vivo. On the basis of our findings, disruption of Smad4 in osteoblasts and osteocytes increased their viability, and this was mediated via the mitochondrial integrity pathway. The 
expression of $\mathrm{Bax} / \mathrm{Bcl}-2$, the amount of cleaved caspase 3 , and the number of TUNEL-positive cells were reduced in Smad $4 \Delta^{O s}$ mice, as was the number of primary calvarial osteoblasts isolated from Smad4 $\Delta^{\mathrm{Os}}$ mice. In addition, apoptosis was increased by osteoblast differentiation in mature osteoblast cells with overexpressed Smad4. Previously, it was reported that Smad-dependent BMP signaling induces osteoblast apoptosis via the mitochondrial pathway in mature osteoblast cells, ${ }^{22}$ although there is some controversy about this. Osx-Cre-mediated Smad4-disrupted mice exhibited increased apoptosis of osteoblasts in bone. ${ }^{16}$ However, because this previous study used Osx-Cre, which acts in precursor osteoblasts, it showed diverse results in the deletion of Smad4 in mice. In the current study, we used Colla1-Cre, which acts in mature osteoblasts and osteocytes. The apoptotic function of Smad4 in mature osteoblasts in vivo is consistent with in vitro data and is also supported by the findings of previous reports. Eliseev et al. ${ }^{36}$ showed that the effect of BMP2 on increasing Bax expression and apoptosis is Runx2-dependent. Furthermore, Runx2 directly binds to the bax promoter and transactivates the bax gene promoter in the Saos2 cell line. Because BMP/Smad induces the expression of Runx $2,37,38$ a finding consistent with our study, the BMP/Smad signaling pathway may affect apoptosis via the mitochondrial integrity pathway. Future work will focus on elucidating detailed mechanisms by which loss of Smad4 in osteoblasts impacts the mitochondrial integrity pathway.

Osteoblast apoptosis, such as steroid-induced apoptosis and microgravity-induced apoptosis, stimulates osteoclastogenesis and bone resorption. These events are regulated by RANKL and OPG secretion from osteoblasts. ${ }^{7}$ Previously, osteocytespecific apoptosis was found to be associated with increased RANKL expression. Furthermore, osteocyte-ablated mice are resistant to bone loss in unloading. ${ }^{8}$ In addition, osteocytespecific disruption of RANKL prevented unloading-induced bone loss. ${ }^{9}$ These reports suggest that osteoblast/osteocyte apoptosis has a key role in orchestrating bone homeostasis. Osteoblast/osteocyte apoptosis, which is associated with increased RANKL expression, may control local osteoclast generation, thereby mediating bone resorption. In our study, bone cell viability increased in Smad44 ${ }^{O s}$ mice, whereas bone resorption decreased with reduced RANKL expression. In addition, Smad $4 \Delta^{O s}$ mice resisted bone loss caused by unloading-induced apoptosis because of the enhanced viability of their osteoblasts and osteocytes. These results suggest that Smad4 may be required for bone homeostasis that is mediated through the regulation of bone cell viability.

\section{CONFLICT OF INTEREST}

The authors declare no conflict of interest.

\section{ACKNOWLEDGEMENTS}

This work was supported by the Basic Science Research Program through the National Research Foundation of Korea (NRF) funded by the Ministry of Science, ICT \& Future Planning, Korea (no. 2013R1A2A1A01007642), and was also supported by the Medical Research Center Program (no. 2008-0062279).

1 Karsenty G, Wagner EF. Reaching a genetic and molecular understanding of skeletal development. Dev Cell 2002; 2: 389-406.

2 Parfitt AM. Osteonal and hemi-osteonal remodeling: the spatial and temporal framework for signal traffic in adult human bone. J Cell Biochem 1994; 55: 273-286.

3 Teitelbaum SL, Ross FP. Genetic regulation of osteoclast development and function. Nat Rev Genet 2003; 4: 638-649.

4 Noble BS, Stevens H, Loveridge N, Reeve J. Identification of apoptotic changes in osteocytes in normal and pathological human bone. Bone 1997; 20: 273-282.

5 Baron R, Kneissel M. WNT signaling in bone homeostasis and disease: from human mutations to treatments. Nat Med 2013; 19: 179-192.

6 Parfitt AM. Targeted and nontargeted bone remodeling: relationship to basic multicellular unit origination and progression. Bone 2002; 30: 5-7.

7 Rucci N, Rufo A, Alamanou M, Teti A. Modeled microgravity stimulates osteoclastogenesis and bone resorption by increasing osteoblast RANKL/OPG ratio. J Cell Biochem 2007; 100: 464-473.

8 Tatsumi S, Ishii K, Amizuka N, Li M, Kobayashi T, Kohno K et al. Targeted ablation of osteocytes induces osteoporosis with defective mechanotransduction. Cell Metab 2007; 5: 464-475.

9 Xiong J, Onal M, Jilka RL, Weistein RS, Manolagas SC, O'Brien CA. Matrix-embedded cells control osteoclast formation. Nat Med 2011; 17: 1235-1241.

10 Jilka RL, Noble B, Weinstein RS. Osteocyte apoptosis. Bone 2013; 54: 264-271.

11 Mann V, Huber C, Kogianni G, Jones D, Noble B. The influence of mechanical stimulation on osteocyte apoptosis and bone viability in human trabecular bone. J Musculoskelet Neuronal Interact 2006; 6: 408-417.

12 Bonewald LF. The amazing osteocyte. J Bone Miner Res 2011; 26: 229-238.

13 Tan X, Weng T, Zhang J, Wang J, Li W, Wan H et al. Smad4 is required for maintaining normal murine postnatal bone homeostasis. J Cell Sci 2007; 120: 2162-2170.

14 Chen G, Deng C, Li YP. TGF- $\beta$ and BMP signaling in osteoblast differentiation and bone formation. Int J Biol Sci 2012; 8: 272-288.

15 Kamiya N, Ye L, Kobayashi T, Mochida Y, Yamauchi M, Kronenberg HM et al. BMP signaling negatively regulates bone mass through sclerostin by inhibiting the canonical Wnt pathway. Development 2008; 135: 3801-3811.

16 Salazar VS, Zarkadis N, Huang L, Norris J, Grimston SK, Mbalaviele G et al. Embryonic ablation of osteoblast Smad4 interrupts matrix synthesis in response to canonical Wnt signaling and causes an osteogenesisimperfecta-like phenotype. J Cell Sci 2013; 126: 4974-4984.

17 Salazar VS, Zarkadis N, Huang L, Watkins M, Kading J, Boner S et al. Postnatal ablation of osteoblast Smad4 enhances proliferative responses to canonical Wnt signaling through interactions with $\beta$-catenin. J Cell Sci 2013; 126: 5598-5609.

18 Kolek O, Gajkowska B, Godlewski MM, Tomasz M. Co-localization of apoptosis-regulating proteins in mouse mammary epithelial HC11 cells exposed to TGF- $\beta 1$. Eur J Cell Biol 2003; 82: 303-312.

19 Lee JH, Wan XH, Song J, Kang JJ, Chung WS, Lee EH et al. TGF- $\beta$-induced apoptosis and reduction of $\mathrm{Bcl}-2$ in human lens epithelial cells in vitro. Curr Eye Res 2002; 25: 147-153.

20 Francis JM, Heyworth CM, Spoonce E, Pierce A, Dexter TM, Whetton AD. Transforming growth factor- $\beta 1$ induces apoptosis independently of $p 53$ and selectively reduces expression of $\mathrm{Bcl}-2$ in multipotent hematopoietic cells. J Biol Chem 2000; 275: 39137-39145.

21 Zunfu K, Xiaowen Z, Lanlan M, Liantang W. Deleted in pancreatic carcinoma locus 4/Smad4 participates in the regulation of apoptosis by affecting the $\mathrm{Bcl}-2 / \mathrm{Bax}$ balance in non-small cell lung cancer. Hum Pathol 2008; 39: 1438-1445.

22 Hyzy SL, Olivares-Navarrete R, Schwartz Z, Boyan BD. BMP2 induces osteoblast apoptosis in a maturation state and Noggin-dependent manner. J Cell Biochem 2012; 113: 3236-3245.

23 Yang X, Li C, Herrera PL, Deng CX. Generation of Smad4/Dpc4 conditional knockout mice. Genesis 2002; 32: 80-81. 
24 Baek WY, Lee MN, Jung JW, Kim SY, Akiyama H, de Crombrugghe B et al. Positive regulation of adult bone formation by osteoblast-specific transcription factor osterix. J Bone Miner Res 2009; 24: 1055-1065.

25 Soriano P. Generalized lacZ expression with the ROSA26 Cre reporter strain. Nat Genet 1999; 21: 70-71.

$26 \mathrm{Kim} \mathrm{TH}$, Bae $\mathrm{CH}$, Lee JC, Ko SO, Yang X, Jiang R et al. B-catenin is required in odontoblasts for tooth root formation. J Dent Res 2013; 92 : 215-221.

27 Meleti Z, Shapiro IM, Adams CS. Inorganic phosphate induces apoptosis of osteoblast-like cells in culture. Bone 2000; 27: 359-366.

28 Chaves Neto AH, Machado D, Yano CL, Ferreira CV. Antioxidant defense and apoptotic effectors in ascorbic acid and $\beta$-glycerophosphate-induced osteoblastic differentiation. Dev Growth Differ 2011; 53: 88-96.

29 Chen J, Shi Y, Regan J, Karuppaiah K, Ornitz DM, Long F. Osx-Cre targets multiple cell types besides osteoblast lineage in postnatal mice. PLOS ONE 2014; 9: e85161.

30 Wang L, Mishina Y, Liu F. Osterix-Cre transgene causes craniofacial bone development defect. Calcif Tissue Int 2015; 96: 129-137.

31 Davey RA, Clarke MV, Sastra S, Skinner JP, Chiang C, Anderson PH et al. Decreased body weight in young Osterix-Cre transgenic mice results in delayed cortical bone expansion and accrual. Transgenic Res 2012; 12: 885-893.

32 Huang W, Olsen BR. Skeletal defects in Osterix-Cre transgenic mice. Transgenic Res 2015; 24: 167-172.

33 Kamiya N, Ye L, Kobayashi T, Lucas DJ, Mochida Y, Yamauchi M et al. Disruption of BMP signaling in osteoblasts through type IA receptor (BMPRIA) increases bone mass. J Bone Miner Res 2008; 23 : 2007-2017.
34 Qiu T, Wu X, Zhang F, Clemens TL, Wan M, Cao X. TGF- $\beta$ type II receptor phosphorylates PTH receptor to integrate bone remodelling signaling. Nat Cell Biol 2010; 12: 224-234.

35 Filvaroff E, Erlebacher A, Ye J, Gitelman SE, Lotz J, Heilman M et al. Inhibition of TGF- $\beta$ receptor signaling in osteoblasts leads to decreased bone remodeling and increased trabecular bone mass. Development 1999; 126: 4267-4279.

36 Eliseev RA, Dong YF, Sampson E, Zuscik MJ, Schwarz EM, O'Keefe RJ et al. Runx2-mediated activation of the Bax gene increases osteosarcoma cell sensitivity to apoptosis. Oncogene 2008; 27: 3605-3614.

37 Ducy P, Zhang R, Geoffroy V, Ridall AL, Karsenty G. Osf2/Cbfa1: a transcriptional activator of osteoblast differentiation. Cell 1997; 89: 747-754.

38 Komori T. Requisite roles of Runx2 and $\mathrm{Cbfb}$ in skeletal development. J Bone Miner Metab 2003; 21: 193-197.

(c) (i) () $\Theta$ This work is licensed under a Creative Commons Attribution-NonCommercial-NoDerivs 4.0 International License. The images or other third party material in this article are included in the article's Creative Commons license, unless indicated otherwise in the credit line; if the material is not included under the Creative Commons license, users will need to obtain permission from the license holder to reproduce the material. To view a copy of this license, visit http://creativecommons.org/licenses/by-nc-nd/4.0/

Supplementary Information accompanies the paper on Experimental \& Molecular Medicine website (http://www.nature.com/emm) 